\title{
LINEARIZATION COEFFICIENTS FOR SHEFFER POLYNOMIAL SETS VIA LOWERING OPERATORS
}

\author{
Y. BEN CHEIKH AND H. CHAGGARA
}

Received 16 May 2005; Revised 2 March 2006; Accepted 12 March 2006

The lowering operator $\sigma$ associated with a polynomial set $\left\{P_{n}\right\}_{n \geq 0}$ is an operator not depending on $n$ and satisfying the relation $\sigma P_{n}=n P_{n-1}$. In this paper, we express explicitly the linearization coefficients for polynomial sets of Sheffer type using the corresponding lowering operators. We obtain some well-known results as particular cases.

Copyright (c) 2006 Hindawi Publishing Corporation. All rights reserved.

\section{Introduction}

Let $\mathscr{P}$ be the linear space of polynomials with complex coefficients. A polynomial sequence $\left\{P_{n}\right\}_{n \geq 0}$ in $\mathscr{P}$ is called a polynomial set if and only if $\operatorname{deg} P_{n}=n$ for all nonnegative integers $n$. Given two polynomial sets $\left\{S_{n}\right\}_{n \geq 0}$ and $\left\{P_{n}\right\}_{n \geq 0}$, the so-called connection problem between them asks to find the coefficients $C_{m}(n)$ in the expression:

$$
S_{n}(x)=\sum_{m=0}^{n} C_{m}(n) P_{m}(x),
$$

which for $S_{n}(x)=x^{n}$ is known as the inversion problem for the polynomial set $\left\{P_{n}\right\}_{n \geq 0}$. When $S_{i+j}(x)=Q_{i}(x) R_{j}(x)$ in $(1.1),\left\{Q_{n}\right\}_{n}$ and $\left\{R_{n}\right\}_{n}$ being two polynomial sets, we are faced to the general linearization problem

$$
Q_{i}(x) R_{j}(x)=\sum_{k=0}^{i+j} L_{i j}(k) P_{k}(x) .
$$

Particular case of this problem is the standard linearization or Clebsch-Gordan-type problem

$$
P_{i}(x) P_{j}(x)=\sum_{k=0}^{i+j} L_{i j}(k) P_{k}(x) .
$$

The computation of the connection and linearization coefficients plays an important role

Hindawi Publishing Corporation

International Journal of Mathematics and Mathematical Sciences

Volume 2006, Article ID 54263, Pages 1-15

DOI 10.1155/IJMMS/2006/54263 
in many situations of pure and applied mathematics and also in physical and quantum chemical applications $[32,33]$. In particular, the study of positivity conditions of $L_{i j}(k)$ has received special attention. This property has many important consequences. It gives rise to a convolution structure associated with the polynomial set $\left\{P_{n}\right\}_{n \geq 0}[8,9,19,36]$. Several sufficient conditions for the sign properties to hold have been derived in $[6,7,37$, 38]. The literature on this topic is extremely vast and a wide variety of methods, based on specific properties of the involved polynomials, have been devised for computing the linearization coefficients $L_{i j}(k)$ either in closed form or by means of recursive relations (usually in $k$ ) $[10,26,27]$, exploiting for this purpose several of their specific properties: recurrence relation [24], generating function $[3,7,15,16,22]$, orthogonality weights and Rodrigue's formula [1, 5], inversion formulas [4, 29], and so forth. A combinatorial approach to solve the linearization problems was also given in $[21,28,39]$.

A general method, based on lowering operators, was developed by the authors $[13,14]$ to solve connection problems. The purpose of this work is to show that such a technique can likewise be used to treat linearization problems.

The outline of the paper is as follows. In Section 2, we give a result for a general linearization problem. Then we prove a useful lemma, generalizing the Leibniz formula, to express explicitly the standard linearization coefficients for Sheffer polynomial sets (Theorem 2.6). In Section 3, for practical uses of the main result, we give the standard linearization coefficients for some well-known basic Sheffer polynomial sets. Finally, in Section 4, we apply Theorem 2.6 to orthogonal Sheffer polynomial sets.

\section{Linearization coefficients}

2.1. A general result. Denote by $\Lambda^{(-1)}$ the space of operators $\sigma$ acting on analytic functions that reduce the degree of every polynomial by exactly one and $\sigma(1)=0$.

It was shown, by the first author, that every polynomial set is quasi-monomial [12]. That is to say, there exist a lowering operator $\sigma$ and a raising operator $\tau$, independent of $n$, such that

$$
\sigma\left(P_{n}\right)=n P_{n-1}, \quad \tau\left(P_{n}\right)=P_{n+1}, \quad n=1,2, \ldots
$$

Definition 2.1. Let $\sigma \in \Lambda^{(-1)}$ and let $\left\{P_{n}\right\}_{n \geq 0}$ be a polynomial set. $\left\{P_{n}\right\}_{n \geq 0}$ is called a $\sigma$ Appell polynomial set if and only if

$$
\sigma\left(P_{n}\right)=n P_{n-1}, \quad n=1,2, \ldots
$$

Definition 2.2. Let $\sigma \in \Lambda^{(-1)}$. A polynomial set $\left\{B_{n}\right\}_{n \geq 0}$ is called the sequence of basic polynomials for $\sigma$ if and only if

(i) $\left\{B_{n}\right\}_{n \geq 0}$ is a $\sigma$-Appell polynomial set;

(ii) $B_{n}(0)=\delta_{0, n}, n=0,1, \ldots$.

In [11], it was shown that every $\sigma \in \Lambda^{(-1)}$ has a unique sequence of basic polynomials. 
Theorem 2.3 [11]. Let $\left\{P_{n}\right\}_{n \geq 0}$ be a polynomial set. Then there exist a unique $\sigma \in \Lambda^{(-1)}$ and a unique power series $A(t)=\sum_{k=0}^{\infty} a_{n} t^{n}, a_{0} \neq 0$, such that $\left\{P_{n}\right\}_{n \geq 0}$ is a $\sigma$-Appell polynomial set and

$$
A(\sigma)\left(B_{n}\right)=P_{n}, \quad n=0,1, \ldots
$$

where $\left\{B_{n}\right\}_{n \geq 0}$ is the sequence of basic polynomials for $\sigma$.

Call $\left\{P_{n}\right\}_{n \geq 0}$ a $\sigma$-Appell polynomial set of transfer power series A.

A $\sigma$-Appell polynomial set of transfer power series $A$ is generated by

$$
G(x, t)=A(t) G_{0}(x, t)=\sum_{n=0}^{\infty} \frac{P_{n}(x)}{n !} t^{n},
$$

where $G_{0}(x, t)$ is a solution of the system

$$
\sigma G_{0}(x, t)=t G_{0}(x, t), \quad G_{0}(x, 0)=1,
$$

and conversely.

Let $\mathscr{P}^{\prime}$ be the algebraic dual of $\mathscr{P}$. We denote by $\langle\mathscr{L}, f\rangle$ the effect of the functional $\mathscr{L} \in \mathscr{P}^{\prime}$ on the polynomial $f \in \mathscr{P}$. Let $\left\{P_{n}\right\}_{n \geq 0}$ be a polynomial set. Its dual sequence $\left\{\mathbb{P}_{n}\right\}_{n \geq 0}$ is defined by

$$
\left\langle\mathbb{P}_{n}, P_{m}\right\rangle=\delta_{n, m}, \quad n, m \geq 0
$$

When $\left\{P_{n}\right\}_{n \geq 0}$ is a $\sigma$-Appell polynomial set of transfer power series $A$, an explicit expression of its dual sequence was given in [11] by

$$
\left\langle\mathbb{P}_{n}, f\right\rangle=\left.\frac{1}{n !} \sigma^{n} \widehat{A}(\sigma)(f)(x)\right|_{x=0}, \quad n=0,1, \ldots, f \in \mathscr{P},
$$

where $\widehat{A}(t)=1 / A(t)$.

Combining (1.2), (2.6), and (2.7), we state the following general result.

Theorem 2.4. Let $\sigma \in \Lambda^{(-1)}$ and $\left\{P_{n}\right\}_{n \geq 0}$ be a $\sigma$-Appell polynomial set of transfer power series $A$. Then the general linearization coefficients in (1.2) are given by

$$
L_{i j}(k)=\frac{1}{k !} \sigma^{k} \widehat{A}(\sigma)\left(Q_{i} R_{j}\right)(0), \quad i, j=0,1, \ldots, k=0,1, \ldots, i+j .
$$

Next, in this paper, we limit ourselves to standard case for Sheffer polynomial sets case.

2.2. Sheffer polynomials. Recall that a polynomial set $\left\{P_{n}\right\}_{n \geq 0}$ is said to be of Sheffer type $A$-zero (Sheffer polynomial set, for shorter,) if and only if it has a generating function of the form $[25,30]$

$$
A(t) \exp (x C(t))=\sum_{n=0}^{\infty} \frac{P_{n}(x)}{n !} t^{n}
$$


4 Linearization coefficients for Sheffer polynomial sets

where $A$ and $C$ are two formal power series:

$$
A(t)=\sum_{k=0}^{\infty} a_{k} t^{k}, \quad a_{0} \neq 0, \quad C(t)=\sum_{k=0}^{\infty} c_{k} t^{k+1}, \quad c_{0} \neq 0 .
$$

It was shown in [12] that a Sheffer polynomial set generated by (2.9) is $\sigma$-Appell polynomial set of transfer power series $A$, where $\sigma=C^{*}(D), D=d / d x$, and $C^{*}$ is the inverse of $C$; that is, $C^{*}(C(t))=C\left(C^{*}(t)\right)=t$.

If $C(t)=t$, we have $\sigma=D$. That corresponds to Appell polynomial sets [2].

In order to apply (2.8) to Sheffer polynomial sets we need the following.

Lemma 2.5 (generalized Leibniz formula). Let $\sigma \in \Lambda^{(-1)}$ and let $\left\{B_{n}\right\}_{n \geq 0}$ be the sequence of basic polynomials for $\sigma$. Suppose that $\sigma$ commutates with the derivative operator $D$. Let $f$ and $g$ be two formal power series. Then,

$$
\sigma^{n}(f(z) g(z))=\sum_{m=n}^{\infty} \sum_{k=0}^{m} c_{k, m}(n) \sigma^{k} f(z) \sigma^{m-k} g(z),
$$

where

$$
c_{k, m}(n)=\left.\frac{1}{k !(m-k) !} \sigma^{n}\left(B_{k} B_{m-k}\right)\right|_{x=0} .
$$

Proof. Let $z_{0} \in \mathbb{C}$. Let us define the translation operators $T_{z_{0}}$ by $T_{z_{0}} f(z)=e^{z_{0} D} f(z)=$ $f\left(z+z_{0}\right)$. Since $\sigma$ commutates with $T_{z_{0}}, f$ and $g$ have the formal power expansions:

$$
f(z)=T_{z_{0}} f\left(z-z_{0}\right)=\sum_{m=0}^{\infty} \frac{\sigma^{m} f\left(z_{0}\right)}{m !} B_{m}\left(z-z_{0}\right), \quad g(z)=\sum_{m=0}^{\infty} \frac{\sigma^{m} g\left(z_{0}\right)}{m !} B_{m}\left(z-z_{0}\right),
$$

by virtue of (2.7). So

$$
\sigma^{n}(f(z) g(z))=\sum_{m=n}^{\infty} \sum_{k=0}^{m} \frac{\sigma^{k} f\left(z_{0}\right)}{k !} \frac{\sigma^{m-k} g\left(z_{0}\right)}{(m-k) !} \sigma^{n}\left(B_{k}\left(z-z_{0}\right) B_{m-k}\left(z-z_{0}\right)\right),
$$

since $\sigma^{n}\left(B_{k}\left(z-z_{0}\right) B_{m-k}\left(z-z_{0}\right)\right)=0$ if $m<n$. Put $z=z_{0}$ in (2.14), we deduce (2.11) since $z_{0}$ is arbitrary.

For the particular case $\sigma=D$, the corresponding basic sequence is $B_{n}(x)=x^{n}$. Then the coefficients in (2.12) are given by

$$
c_{k, m}(n)=\left.\frac{1}{k !(m-k) !} D^{n}\left(x^{m}\right)\right|_{x=0}=\frac{n !}{k !(m-k) !} \delta_{n, m}
$$

and (2.11) is reduced to the well-known Leibniz formula

$$
D^{n}(f(z) g(z))=\sum_{k=0}^{n}\left(\begin{array}{l}
n \\
k
\end{array}\right) D^{k} f(z) D^{n-k} g(z) .
$$


As every Sheffer polynomial set generated by (2.9) may be viewed as a $\sigma$-Appell polynomial set of transfer power series $A$ where $\sigma=C^{*}(D)$, we use this property to state our following main result.

Theorem 2.6. The linearization coefficients in (1.3) with $\left\{P_{n}\right\}_{n \geq 0}$ a Sheffer polynomial set generated by (2.9) are given by

$$
L_{i j}(k)=\left.\sum_{m \geq k} \sum_{p=0}^{m}\left(\begin{array}{c}
i \\
p
\end{array}\right)\left(\begin{array}{c}
j \\
m-p
\end{array}\right) l_{p, m-p}(k) \hat{A}\left(C^{*}(D)\right)\left(P_{i-p} P_{j-m+p}(x)\right)\right|_{x=0},
$$

where $l_{n m}(k)$ are the standard linearization coefficients for the corresponding basic sequence generated by

$$
\frac{1}{k !} C^{* k}(C(t)+C(s))=\sum_{n, m} \frac{l_{n m}(k)}{n ! m !} t^{n} s^{m} .
$$

Proof. $\left\{P_{n}\right\}_{n \geq 0}$ is a $\sigma$-Appell polynomial set of transfer power series $A$, where $\sigma=C^{*}(D)$. Then by virtue of Theorem 2.4 and (2.11) we derive (2.17).

The basic sequence $\left\{B_{n}\right\}_{n \geq 0}$ is a $\sigma$-Appell polynomial set of transfer power series 1 . So according to (2.8) and (2.16), we have

$$
\begin{aligned}
l_{i j}(k) & =\left.\frac{1}{k !} \sigma^{k}\left(B_{i} B_{j}\right)\right|_{x=0}=\left.\frac{1}{k !} C^{* k}(D)\left(B_{i} B_{j}\right)\right|_{x=0} \\
& =\left.\frac{1}{k !} \sum_{n \geq k} \alpha_{n, k} D^{k}\left(B_{i} B_{j}\right)\right|_{x=0}, \quad \text { where } C^{* k}(t)=\sum_{n \geq k} \alpha_{n, k} t^{n}, \\
& =\left.\frac{1}{k !} \sum_{n \geq k} \alpha_{n, k}\left(\sum_{p=0}^{n}\left(\begin{array}{l}
n \\
p
\end{array}\right) D^{p} B_{i} D^{k-p} B_{j}\right)\right|_{x=0} \\
& =\left.\frac{1}{k !} \sum_{n \geq k} \alpha_{n, k}\left(\sum_{p=0}^{n}\left(\begin{array}{l}
n \\
p
\end{array}\right) C(\sigma)^{p} B_{i} C(\sigma)^{k-p} B_{j}\right)\right|_{x=0} \\
& =\left.\frac{1}{k !} \sum_{n \geq k} \alpha_{n, k}\left(C(\sigma)\left(B_{i}\right)+C(\sigma)\left(B_{j}\right)\right)^{n}\right|_{x=0} \\
& =\frac{1}{k !} C^{* k}\left(C\left(\sigma\left(B_{i}\right)\right)+\left.C\left(\sigma\left(B_{j}\right)\right)\right|_{x=0} .\right.
\end{aligned}
$$

Put $(1 / k !) C^{* k}(C(t)+C(s))=\sum_{n, m} a_{n, m}(k) t^{n} s^{m}$. It follows from (2.19) and Definition 2.2 that

$$
\begin{aligned}
l_{i, j}(k) & =\left.\frac{1}{k !} \sum_{n, m} a_{n, m}(k) \sigma^{n}\left(B_{i}\right) \sigma^{m}\left(B_{j}\right)\right|_{x=0} \\
& =\left.\frac{1}{k !} \sum_{n, m} a_{n, m}(k) \frac{i !}{(i-n) !} B_{i-n}(0) \frac{j !}{(j-m) !} B_{j-m}(0)\right|_{x=0}=i ! j ! a_{i, j}(k),
\end{aligned}
$$

which gives (2.18). 


\section{Linearization coefficients for Sheffer polynomial sets}

Remark 2.7. A similar proof may be used to express general linearization coefficients in (1.2) where the involved three polynomial sets are of Sheffer type.

Next, in Section 3, we use (2.18) to express explicitly the standard linearization coefficients for some well-known basic Sheffer polynomial sets. Then, in Section 4, in order to show the efficiency of the proposed approach, we apply Theorem 2.6 to orthogonal Sheffer polynomial sets to derive some already obtained results in the literature by alternative methods.

\section{Linearization coefficients for basic polynomials}

3.1. Stirling polynomials. The Stirling polynomial set $\left\{x^{[n]}=x(x-1) \cdots(x-n+1)\right\}_{n \geq 0}$ is generated by

$$
(1+t)^{x}=\sum_{n=0}^{\infty} \frac{x^{[n]}}{n !} t^{n}
$$

For this case we have $C(t)=\log (1+t)$ and $C^{*}(t)=e^{t}-1$.

It follows that $\left\{x^{[n]}\right\}_{n \geq 0}$ is a $\Delta$-Appell polynomial set, where $\Delta=e^{D}-1$ is the difference operator, and

$$
\begin{aligned}
\frac{1}{k !} C^{* k}(C(t)+C(s)) & =\frac{1}{k !}(t+s+s t)^{k}=\sum_{n, m} \frac{t^{m+k-n} s^{k-m}}{(k-n) !(n-m) ! m !} \\
& =\sum_{i, j} \frac{t^{i} s^{j}}{(k-j) !(k-i) !(i+j-k) !} .
\end{aligned}
$$

Then

$$
x^{[i]} x^{[j]}=\sum_{k=0}^{\min (i, j)} k !\left(\begin{array}{l}
i \\
k
\end{array}\right)\left(\begin{array}{l}
j \\
k
\end{array}\right) x^{[i+j-k]} .
$$

According to (3.3), the following relations can be derived

$$
\begin{aligned}
\left(\begin{array}{l}
x \\
n
\end{array}\right)\left(\begin{array}{c}
x \\
m
\end{array}\right) & =\sum_{k=0}^{\min (n, m)} \frac{(n+m-k) !}{(n-k) !(m-k) ! k !}\left(\begin{array}{c}
x \\
n+m-k
\end{array}\right), \\
(x)_{n}(x)_{m} & =\sum_{k=0}^{\min (n, m)}(-1)^{k} k !\left(\begin{array}{l}
n \\
k
\end{array}\right)\left(\begin{array}{l}
m \\
k
\end{array}\right)(x)_{n+m-k},
\end{aligned}
$$

where $(x)_{n}=x(x+1) \cdots(x+n-1)$.

Also, from (3.3), one can see that (2.11) contains as a particular case the well-known Jordan formula [20]

$$
\Delta^{n}(f g)(z)=\sum_{k=0}^{n}\left(\begin{array}{l}
n \\
k
\end{array}\right) \Delta^{k} f(z) \Delta^{n-k} g(z+k) .
$$


In fact, for the special case $\sigma=\Delta$, the corresponding basic sequence is $B_{n}(x)=x^{[n]}$. Then (2.12) is reduced to

$$
c_{k, m}(n)=\frac{n !}{k !(m-k) !} l_{k, m-k}(n)=\frac{n !}{(n-k) !(n-m) !(m+k-n) !} .
$$

It follows from (2.11) and (3.6) that

$$
\begin{aligned}
\Delta^{n}(f(z) g(z)) & =\sum_{m=0}^{\infty} \sum_{k=0}^{m} c_{k, m} \Delta^{k} f(z) \Delta^{m-k} g(z)=\sum_{k=0}^{\infty} \frac{n !}{k !} \Delta^{k} f(z)\left(\sum_{m=0}^{\infty} \frac{l_{k, m}(n)}{m !} \Delta^{m} g(z)\right) \\
& =\sum_{k=0}^{\infty} \Delta^{k} f(z)\left(\sum_{m=0}^{\infty} \frac{n !}{(n-k) !(n-m) !(m+k-n) !} \Delta^{m} g(z)\right) \\
& =\sum_{k=0}^{\infty}\left(\begin{array}{l}
n \\
k
\end{array}\right) \Delta^{k} f(z) \Delta^{n-k}\left(\sum_{p=0}^{\infty}\left(\begin{array}{l}
k \\
p
\end{array}\right) \Delta^{p} g(z)\right),
\end{aligned}
$$

which, in view of the expansion formula [20]

$$
g(z+a)=\sum_{p=0}^{\infty}\left(\begin{array}{l}
a \\
p
\end{array}\right) \Delta^{p} g(z),
$$

gives (3.5).

3.2. Basic Laguerre polynomials. The basic Laguerre polynomials $\left\{L_{n}(x)\right\}_{n \geq 0}$ are generated by

$$
e^{x(t /(t-1))}=\sum_{n=0}^{\infty} L_{n}(x) t^{n} .
$$

Then $C(t)=C^{*}(t)=t /(t-1)$. It follows that $\left\{n ! L_{n}(x)\right\}_{n \geq 0}$ is a $\sigma$-Appell polynomial set, where $\sigma=D /(D-1)$ is the Laguerre operator.

For this case we have

$$
\begin{aligned}
\frac{1}{k !} C^{* k}(C(t)+C(s)) & =\frac{1}{k !}\left(\frac{t+s-2 s t}{1-s t}\right)^{k} \\
& =\sum_{i, j} \sum_{n \geq 0} \frac{(k)_{n}(-2)^{i+j-k-2 n}}{(n+k-j) !(n+k-i) !(i+j-k-2 n) !} t^{i} s^{j} .
\end{aligned}
$$

Then

$$
\begin{aligned}
l_{i j}(k) & =\sum_{n \geq 0} \frac{(k)_{n} k !(-2)^{i+j-k-2 n}}{n !(n+k-i) !(n+k-j) !(i+j-k-2 n) !} \\
& =\frac{(-2)^{i+j-k} k !}{(i+j-k) !(k-i) !(k-j) !}{ }_{3} F_{2}\left(k, \frac{k-i-j}{2}, \frac{k-i-j+1}{2} ; 1\right) .
\end{aligned}
$$


8 Linearization coefficients for Sheffer polynomial sets

3.3. Basic Meixner polynomials. The basic Meixner polynomial set $\left\{P_{n}(x)\right\}_{n \geq 0}$ is generated by

$$
e^{x \ln ((1-t / a) /(1-t))}=\sum_{n=0}^{\infty} \frac{P_{n}(x)}{n !} t^{n} .
$$

For this case we have

$$
C(t)=\ln \left(\frac{1-t / a}{1-t}\right), \quad C^{*}(t)=\frac{e^{t}-1}{e^{t}-1 / a}
$$

It follows that $\left\{P_{n}(x)\right\}_{n \geq 0}$ is a $\sigma$-Appell polynomial set, where $\sigma=\left(e^{D}-1\right) /\left(e^{D}-1 / a\right)$, and

$$
\begin{aligned}
\frac{1}{k !} C^{* k}(C(t)+C(s)) & =\frac{1}{k !}\left(\frac{t+s-(1+1 / a) s t}{1-s t / a}\right)^{k} \\
& =\sum_{i, j} \sum_{n \geq 0} \frac{(k)_{n}(-1-1 / a)^{i+j+k-2 n}}{n ! a^{n}(n+k-j) !(n+k-i) !(i+j-k-2 n) !} t^{i} s^{j} .
\end{aligned}
$$

Then

$$
\begin{aligned}
l_{i j}(k) & =\sum_{n \geq 0} \frac{(k)_{n} i ! j !(-1-1 / a)^{i+j-k-2 n}}{n ! a^{n}(n+k-i) !(n+k-j) !(i+j-k-2 n) !} \\
& =\frac{i ! j !(-1-1 / a)^{i+j-k}}{(i+j-k) !(k-i) !(k-j) !}{ }_{3} F_{2}\left(k, \frac{k-i-j}{2}, \frac{k-i-j+1}{2} ; \frac{4 a}{(a+1)^{2}}\right) .
\end{aligned}
$$

3.4. Basic Meixner-Pollaczek polynomials. The basic Meixner-Pollaczek polynomial set $\left\{P_{n}(x)\right\}_{n \geq 0}$ is generated by

$$
e^{x \arctan (t /(1+\delta t))}=\sum_{n=0}^{\infty} \frac{P_{n}(x)}{n !} t^{n} .
$$

For this case we have

$$
C(t)=\arctan \frac{t}{1+\delta t}, \quad C^{*}(t)=\frac{\tan t}{1-\delta \tan t} .
$$

It follows that $\left\{P_{n}(x)\right\}_{n \geq 0}$ is a $\sigma$-Appell polynomial set, where $\sigma=\tan D /(1-\delta \tan D)$ and

$$
\begin{aligned}
\frac{1}{k !} C^{* k}(C(t)+C(s)) & =\frac{1}{k !}\left(\frac{t+s+2 \delta s t}{1-\left(1+\delta^{2}\right) s t}\right)^{k} \\
& =\sum_{i, j} \sum_{n \geq 0} \frac{(k)_{n}\left(1+\delta^{2}\right)^{n}(2 \delta)^{i+j-k-2 n}}{n !(n+k-j) !(n+k-i) !(i+j-k-2 n) !} t^{i} s^{j} .
\end{aligned}
$$


Then

$$
\begin{aligned}
l_{i j}(k) & =\sum_{n \geq 0} \frac{(k)_{n}\left(1+\delta^{2}\right)^{n} i ! j !(2 \delta)^{i+j-k-2 n}}{n !(n+k-i) !(n+k-j) !(i+j-k-2 n) !} \\
& =\frac{i ! j !(2 \delta)^{i+j-k}}{(i+j-k) !(k-i) !(k-j) !}{ }_{3} F_{2}\left(\begin{array}{c}
k, \frac{k-i-j}{2}, \frac{k-i-j+1}{2} \\
k-i+1, k-j+1
\end{array} \frac{1+\delta^{2}}{\delta^{2}}\right) .
\end{aligned}
$$

\section{Orthogonal Sheffer polynomials}

Let $\left\{P_{n}\right\}_{n \geq 0}$ be an orthogonal $\sigma$-Appell polynomial set of transfer power series $A$. The linear functional $\mathscr{L}$ for which the orthogonality holds is given by [34]

$$
\langle\mathscr{L}, f\rangle=\widehat{A}(\sigma)(f)(0) .
$$

We use this relation and Theorem 2.6 to state the following.

Corollary 4.1. The linearization coefficients in (1.3) for $\left\{P_{n}\right\}_{n \geq 0}$ an orthogonal $\sigma$-Appell polynomial set of transfer power series $A$ of Sheffer type are given by

$$
L_{i j}(k)=\sum_{2 s \leq i+j-k}\left(\begin{array}{l}
i \\
s
\end{array}\right)\left(\begin{array}{l}
j \\
s
\end{array}\right) l_{i-s, j-s}(k) I_{s},
$$

where $I_{s}=\left\langle\mathscr{L}, P_{s} P_{s}\right\rangle=\left.\widehat{A}(\sigma)\left(P_{s}^{2}\right)(x)\right|_{x=0}$ and $l_{i j}$ are the standard linearization coefficients for the corresponding basic sequence.

An immediate consequence of this result is the following.

Corollary 4.2. A sufficient condition to ensure the positivity of the standard linearization coefficients for an orthogonal Sheffer polynomial set is the positivity of those associated with the corresponding basic sequence.

It follows, from the results obtained in Section 3, that the standard linearization coefficients for Hermite, Charlier, monic Laguerre polynomials $\left\{(-1)^{n} n ! L_{n}^{\alpha}(x)\right\}_{n \geq 0}$, and Meixner-Pollaczek polynomials are positive.

Let us return now to Corollary 4.1 to mention that this result concerns exactly five classes of Sheffer polynomials according to Meixner characterization [17]. In Table 4.1, we recall these classes with the corresponding lowering operators and transfer power series according to our analysis.

Next, for each case, we use (4.2) to express explicitly the corresponding standard linearization coefficients in terms of hypergeometric series.

4.1. Hermite polynomials. The Hermite polynomials $\left\{H_{n}\right\}_{n \geq 0}$ are generated by

$$
e^{-t^{2}} e^{2 x t}=\sum_{n=0}^{\infty} \frac{H_{n}(x)}{n !} t^{n}
$$


10 Linearization coefficients for Sheffer polynomial sets

Table 4.1. Orthogonal Sheffer polynomial sets.

\begin{tabular}{c|cc}
\hline Polynomial set & Lowering operator & Transfer power series \\
\hline $\begin{array}{c}\text { Hermite } \\
H_{n}(\mathrm{x})\end{array}$ & $\sigma=\frac{D}{2}$ & $A(t)=e^{-t^{2}}$ \\
\hline $\begin{array}{c}\text { Charlier } \\
C_{n}^{a}(x), a>0\end{array}$ & $\sigma=\Delta=e^{D}-1$ & $A(t)=e^{-a t}$ \\
\hline $\begin{array}{c}\text { Laguerre } \\
L_{n}^{\alpha}(x), \alpha>-1\end{array}$ & $\sigma=\frac{D}{D-1}$ & $A(t)=(1-t)^{-\alpha-1}$ \\
\hline $\begin{array}{c}\text { Meixner } \\
M_{n}(x, \beta, a), \\
\beta>0,0<a<1\end{array}$ & $\sigma=\frac{\Delta}{(a-1) / a+\Delta}$ & $A(t)=(1-t)^{-\beta}$ \\
\hline $\begin{array}{c}\text { Meixner-Pollaczek } \\
P_{n}(x ; \delta, \eta)\end{array}$ & $\sigma=\frac{\tan D}{1-\delta \tan D}$ & $A(t)=\left[(1+\delta t)^{2}+t^{2}\right]^{-\eta / 2}$ \\
$\delta>0, \eta>0$ & & \\
\hline
\end{tabular}

Then $\sigma=D / 2$ and $A(t)=e^{-t^{2}}$. Since the corresponding basic sequence is $\left\{(2 x)^{n}\right\}_{n \geq 0}$, we get $l_{i j}(k)=\delta_{i+j, k}$. The linear functional for which the orthogonality holds is [34]

$$
\widehat{A}(\sigma)(f)(0)=\exp \left(\sigma^{2}\right)(f)(0)=\frac{1}{\sqrt{\pi}} \int_{-\infty}^{+\infty} e^{-x^{2}} f(x) d x
$$

Then we have [17] $I_{s}=s ! 2^{s}$. According to (4.2), we deduce [18]

$$
L_{i j}(k)= \begin{cases}\left(\begin{array}{l}
i \\
s
\end{array}\right)\left(\begin{array}{l}
j \\
s
\end{array}\right) 2^{s} s ! & \text { if } k=i+j-2 s \\
0 & \text { otherwise }\end{cases}
$$

4.2. Charlier polynomials. The Charlier polynomials $\left\{C_{n}^{a}\right\}_{n \geq 0}$ are generated by

$$
e^{-a t}(1+t)^{x}=\sum_{n=0}^{\infty} \frac{C_{n}^{a}(x)}{n !} t^{n}
$$

Then $\sigma=\Delta$ and $A(t)=e^{-a t}$. Since the corresponding basic sequence is the Stirling polynomial set $\left\{x^{[n]}\right\}_{n \geq 0}$, we have $l_{i j}(k)=k !\left(\begin{array}{c}i \\ i+j-k\end{array}\right)\left(\begin{array}{c}j \\ i+j-k\end{array}\right)$ by virtue of (3.3). The linear functional for which the orthogonality holds is [34]

$$
\widehat{A}(\sigma)(f)(0)=\exp (a \Delta)(f)(0)=e^{-a} \sum_{j=0}^{\infty} \frac{a^{j}}{j !} f(j) .
$$


Then we have [17] $I_{s}=a^{s} s$ !. According to (4.2), we obtain [39]

$$
\begin{aligned}
L_{i j}(k) & =\sum_{s \geq 0} \frac{i ! j ! a^{s}}{(s+k-i) !(s+k-j) !(s) !(i+j-k-2 s) !} \\
& =\frac{i ! j !}{(i+j-k) !(k-i) !(k-j) !}{ }_{2} F_{2}\left(\begin{array}{c}
\frac{k-i-j}{2}, \frac{k-i-j+1}{2} \\
k-i+1, k-j+1
\end{array}\right) .
\end{aligned}
$$

4.3. Laguerre polynomials. The Laguerre polynomials $\left\{L_{n}^{\alpha}\right\}_{n \geq 0}$ are generated by [17]

$$
(1-t)^{-\alpha-1} \exp \left(x \frac{t}{t-1}\right)=\sum_{n=0}^{\infty} L_{n}^{\alpha}(x) t^{n}
$$

Then the lowering and transfer operators for $\left\{n ! L_{n}^{\alpha}\right\}_{n \geq 0}$ are, respectively, $\sigma=D /(D-1)$ and $A(\sigma)=(1-\sigma)^{-\alpha-1}$. The linear functional for which the orthogonality holds is [34]

$$
\widehat{A}(\sigma)(f)(0)=(1-\sigma)^{\alpha+1}(f)(0)=\frac{1}{\Gamma(\alpha+1)} \int_{0}^{+\infty} f(x) x^{\alpha} e^{-x} d x, \quad \alpha>-1 .
$$

For this case we have $[17,23] I_{s}=s !(\alpha+1)_{s}$.

According to (3.11) and (4.2), we deduce the linearization coefficients for $\left\{L_{n}^{\alpha}\right\}_{n \geq 0}$,

$$
L_{i j}(k)=\frac{(-2)^{i+j-k}}{k !} \sum_{n, s} \frac{2^{-2(n+s)}(k)_{n}(\alpha+1)_{s}}{n ! s !((n+s)+k-i) !((n+s)+k-j) !(i+j-k-2(n+s)) !},
$$

which, in view of the well-known relationship [31, 35]:

$\sum_{n, m} \frac{(\rho)_{n}(\sigma)_{m} c_{n+m}}{m ! n !} x^{n+m}=\sum_{n} \frac{(\rho+\sigma)_{n} c_{n}}{n !} x^{n}, \quad\left\{c_{n}\right\}$ being a sequence of complex numbers,

assumes the form [28]

$$
\begin{aligned}
L_{i j}(k) & =\frac{(-2)^{i+j-k}}{k !} \sum_{p \geq 0} \frac{(\alpha+1+k)_{p} 2^{-2 p}}{p !(p+k-i) !(p+k-j) !(i+j-k-2 p) !} \\
& =\frac{(-2)^{i+j-k}}{k !(i+j-k) !(k-i) !(k-j) !}{ }_{3} F_{2}\left(\begin{array}{c}
k+\alpha+1, \frac{k-i-j}{2}, \frac{k-i-j+1}{2} \\
k-i+1, k-j+1
\end{array}\right) .
\end{aligned}
$$

4.4. Meixner polynomials. The Meixner polynomial set $\left\{M_{n}(x ; \beta, a)\right\}_{n \geq 0}$ is generated by [17]

$$
(1-t)^{-\beta}\left(\frac{1-t / a}{1-t}\right)^{x}=\sum_{n=0}^{\infty} M_{n}(x ; \beta, a) \frac{t^{n}}{n !} .
$$


12 Linearization coefficients for Sheffer polynomial sets

It follows that $\sigma=\left(e^{D}-1\right) /\left(e^{D}-1 / a\right)$ and $A(t)=(1-t)^{-\beta}$. The linear functional for which the orthogonality holds is [34]

$$
\widehat{A}(\sigma)(f)(0)=(1-\sigma)^{\beta}(f)(0)=(1-a)^{\beta} \sum_{j=0}^{\infty} \frac{(\beta)_{j}}{j !} a^{j} f(j) .
$$

For this case we have [18] $I_{s}=s !(\beta)_{s} a^{-s}$. According to (3.15), (4.2), and (4.12) we obtain [9]

$$
\begin{aligned}
& L_{i j}(k)=i ! j !\left(-1-\frac{1}{a}\right)^{i+j-k} \sum_{p \geq 0} \frac{(\beta+k)_{p}(1+a)^{-2 p} a^{p}}{p !(p+k-i) !(p+k-j) !(i+j-k-2 p) !} \\
& =\frac{i ! j !(-1-1 / a)^{i+j-k}}{(i+j-k) !(k-i) !(k-j) !}{ }_{3} F_{2}\left(\begin{array}{c}
k+\beta, \frac{k-i-j}{2}, \frac{k-i-j+1}{2} \\
k-i+1, k-j+1
\end{array} \frac{4 a}{(a+1)^{2}}\right) .
\end{aligned}
$$

So, the linearization coefficients alternate in sign just as in the Laguerre polynomial set case.

4.5. Meixner-Pollaczek polynomials. The Meixner-Pollaczek polynomials are generated by [17]

$$
\left[(1+\delta t)^{2}+t^{2}\right]^{-\eta / 2} e^{x \arctan (t /(1+\delta t))}=\sum_{n=0}^{\infty} \frac{P_{n}(x, \delta, \eta)}{n !} t^{n}
$$

Then we have

$$
\sigma=\frac{\tan D}{1-\delta \tan D}, \quad A(t)=\left((1+\delta t)^{2}+t^{2}\right)^{-\eta / 2}
$$

To obtain the effect of the linear functional $\widehat{A}(\sigma)$ on analytic functions, we need the following relation [39]:

$$
\frac{1}{\pi \Gamma(\rho)} \int_{-\infty}^{+\infty} e^{-(\pi-2 t) x}\left|\Gamma\left(\frac{\rho+i x}{2}\right)\right|^{2} d x=(2 \sin t)^{-\rho}, \quad \rho>0
$$

It follows from (4.18) that

$$
\begin{aligned}
\widehat{A}(\sigma(f)) & =(\cos D-\delta \sin D)^{-\eta}(f)=\left(\frac{\sin (\pi / 2+D+\arctan \delta)}{\sin (\pi / 2+\arctan \delta)}\right)^{-\eta}(f) \\
& =\frac{\int_{-\infty}^{+\infty} f(x) \omega(x) d x}{\int_{-\infty}^{+\infty} \omega(x) d x},
\end{aligned}
$$


where $\omega(x)=[\Gamma(\eta / 2)]^{-2}|\Gamma(\eta+i x / 2)|^{2} \exp \left(-x \tan ^{-1} \delta\right)$. So we have $[17,23] I_{s}=\left(\delta^{2}+\right.$ $1)^{s} s !(\eta)_{s}$. According to (3.19), (4.2), and (4.12), we obtain [39]

$$
\begin{aligned}
L_{i j}(k) & =i ! j !(2 \delta)^{i+j-k} \sum_{p \geq 0} \frac{(\eta+k)_{p}(2 \delta)^{-2 p}\left(1+\delta^{2}\right)^{p}}{p !(p+k-i) !(p+k-j) !(i+j-k-2 p) !} \\
& =\frac{i ! j !(2 \delta)^{i+j-k}}{(i+j-k) !(k-i) !(k-j) !}{ }_{3} F_{2}\left(\begin{array}{c}
k+\eta, \frac{k-i-j}{2}, \frac{k-i-j+1}{2} \\
k-i+1, k-j+1
\end{array} \frac{1+\delta^{2}}{\delta^{2}}\right) .
\end{aligned}
$$

Taking $\delta \mapsto 0$ and $\eta=2 \lambda$ in (4.21) and using the well-known relation [35], namely,

$$
\left(\frac{-2 k+1}{2}\right)_{k}=(-1)^{k} \frac{(2 k) !}{2^{2 k} k !}, \quad k=0,1,2, \ldots
$$

we obtain [3]

$$
p_{i}^{(\lambda)}(x) p_{j}^{(\lambda)}(x)=\sum_{k=0}^{\min (i, j)} \frac{\Gamma(i+j-2 k+2 \lambda+1)(i+j-2 k) !}{k !(i-k) !(j-k) !} p_{i+j-2 k}^{(\lambda)}(x),
$$

where $p_{n}^{(\lambda)}(x)=(1 / n !) P_{n}(x ; 0,2 \lambda)$ designates the symmetric Meixner-Pollaczek polynomials.

\section{References}

[1] R. Álvarez-Nodarse, R. J. Yáñez, and J. S. Dehesa, Modified Clebsch-Gordan-type expansions for products of discrete hypergeometric polynomials, Journal of Computational and Applied Mathematics 89 (1998), no. 1, 171-197.

[2] P. Appell, Sur une classe de polynômes, Annales Scientifiques de l'École Normale Supérieure 9 (1880), no. 2, 119-144.

[3] T. K. Araaya, Linearization and connection problems for the symmetric Meixner-Pollaczek polynomials, Uppsala University (2003), 59-70.

[4] I. Area, E. Godoy, A. Ronveaux, and A. Zarzo, Solving connection and linearization problems within the Askey scheme and its q-analogue via inversion formulas, Journal of Computational and Applied Mathematics 133 (2001), no. 1-2, 151-162.

[5] P. L. Artés, J. S. Dehesa, A. Martínez-Finkelshtein, and J. Sánchez-Ruiz, Linearization and connection coefficients for hypergeometric-type polynomials, Journal of Computational and Applied Mathematics 99 (1998), no. 1-2, 15-26.

[6] R. Askey, Orthogonal polynomials and positivity, Studies in Applied Mathematics, Special Functions and Wave Propagation, vol. 6, SIAM, Pennsylvania, 1970, pp. 64-85.

[7] _ Orthogonal Polynomials and Special Functions, CBMS Regional Conference Series, vol. 21, SIAM, Pennsylvania, 1975.

[8] R. Askey and G. Gasper, Linearization of the product of Jacobi polynomials. III, Canadian Journal of Mathematics 23 (1971), 332-338.

[9]_ Convolution structures for Laguerre polynomials, Journal d'Analyse Mathématique 31 (1977), 48-68.

[10] S. Belmehdi, S. Lewanowicz, and A. Ronveaux, Linearization of the product of orthogonal polynomials of a discrete variable, Applicationes Mathematicae 24 (1997), no. 4, 445-455. 
[11] Y. Ben Cheikh, On obtaining dual sequences via quasi-monomiality, Georgian Mathematical Journal 9 (2002), no. 3, 413-422.

[12] Some results on quasi-monomiality, Applied Mathematics and Computation 141 (2003), no. 1, 63-76.

[13] Y. Ben Cheikh and H. Chaggara, Connection problems via lowering operators, Journal of Computational and Applied Mathematics 178 (2005), no. 1-2, 45-61.

[14] Connection coefficients between Boas-Buck polynomial sets, Journal of Mathematical Analysis and Applications 319 (2006), no. 2, 665-689.

[15] L. Carlitz, The product of certain polynomials analogous to the Hermite polynomials, The American Mathematical Monthly 64 (1957), 723-725.

[16] _ Products of Appell polynomials, Collectanea Mathematica 15 (1963), 245-258.

[17] T. S. Chihara, An Introduction to Orthogonal Polynomials, Gordon and Breach, New York, 1978.

[18] A. Erdélyi, W. Magnus, F. Oberhettinger, and F. G. Tricomi, Higher Transcendental Functions. Vols. I, II, McGraw-Hill, New York, 1953.

[19] G. Gasper, Linearization of the product of Jacobi polynomials. I, Canadian Journal of Mathematics $22(1970), 171-175$.

[20] C. Jordan, Calculus of Finite Differences, 2nd ed., Chelsea, New York, 1960.

[21] D. Kim and J. Zeng, A combinatorial formula for the linearization coefficients of general Sheffer polynomials, European Journal of Combinatorics 22 (2001), no. 3, 313-332.

[22] H. Kleindienst and A. Lüchow, Multiplication theorems for orthogonal polynomials, International Journal of Quantum Chemistry 48 (1993), no. 4, 239-247.

[23] R. Koekoek and R. F. Swarrtow, The Askey-scheme of hypergeometric orthogonal polynomials and its q-analogue, Tech. Rep. 98-17, Faculty of the Technical Mathematics and Informatics, Delft University of Technology, Delft, 1998.

[24] C. Markett, Linearization of the product of symmetric orthogonal polynomials, Constructive Approximation 10 (1994), no. 3, 317-338.

[25] E. D. Rainville, Special Functions, Macmillan, New York, 1960.

[26] A. Ronveaux, Orthogonal polynomials: connection and linearization coefficients, Proceedings of the International Workshop on Orthogonal Polynomials in Mathematical Physics (M. Alfano, R. Álvarez-Nodarse, G. López Lagomasino, and F. Marcellán, eds.), Madrid, June 1996.

[27] A. Ronveaux, M. N. Hounkonnou, and S. Belmehdi, Generalized linearization problems, Journal of Physics. A: Mathematical and General 28 (1995), no. 15, 4423-4430.

[28] J. Sánchez-Ruiz, P. L. Artés, A. Martínez-Finkelshtein, and J. S. Dehesa, Linearization problems of hypergeometric polynomials in quantum physics, Proceedings of the Melfi Workshop on Advanced Special Functions and Applications (G. Dattoli, H. M. Srivastava, and D. Cocolicchio, eds.), Rome, May 1999.

[29] J. Sánchez-Ruiz and J. S. Dehesa, Some connection and linearization problems for polynomials in and beyond the Askey scheme, Journal of Computational and Applied Mathematics 133 (2001), no. 1-2, 579-591.

[30] I. M. Sheffer, Some properties of polynomial sets of type zero, Duke Mathematical Journal 5 (1939), no. 3, 590-622.

[31] H. M. Srivastava, On the reducibility of Appell's function $F_{4}$, Canadian Mathematical Bulletin 16 (1973), 295-298.

[32] _ A unified theory of polynomial expansions and their applications involving ClebschGordan type linearization relations and Neumann series, Astrophysics and Space Science 150 (1988), no. 2, 251-266.

[33] __ Some Clebesch-Gordan type linearization relations and other polynomial expansions associated with a class of generalized multiple hypergeometric series arising in physical and quantum chemical applications, Journal of Physics A: Mathematical and General 21 (1988), 4463-4470. 
[34] H. M. Srivastava and Y. Ben Cheikh, Orthogonality of some polynomial sets via quasi-monomiality, Applied Mathematics and Computation 141 (2003), no. 2-3, 415-425.

[35] H. M. Srivastava and H. L. Manocha, A Treatise on Generating Functions, John Willey \& Sons, New York; Brisbane, Toronto, 1984.

[36] R. Szwarc, Convolution structures associated with orthogonal polynomials, Journal of Mathematical Analysis and Applications 170 (1992), no. 1, 158-170.

[37] _ Nonnegative linearization and quadratic transformation of Askey-Wilson polynomials, Canadian Mathematical Bulletin 39 (1996), no. 2, 241-249.

[38] _ A necessary and sufficient condition for nonnegative product linearization of orthogonal polynomials, Constructive Approximation 19 (2003), no. 4, 565-573.

[39] J. Zeng, Weighted derangements and the linearization coefficients of orthogonal Sheffer polynomials, Proceedings of the London Mathematical Society. Third Series 65 (1992), no. 1, 1-22.

Y. Ben Cheikh: Département de Mathématiques, Faculté des Sciences de Monastir, Université de Monastir, 5019 Monastir, Tunisia

E-mail address: youssef.bencheikh@planet.tn

H. Chaggara: Département de Préparation en Math-Physique, Institut Préparatoire aux Études d’Ingénieur de Monastir, 5019 Monastir, Tunisia

E-mail address: hamza.chaggara@ipeim.rnu.tn 


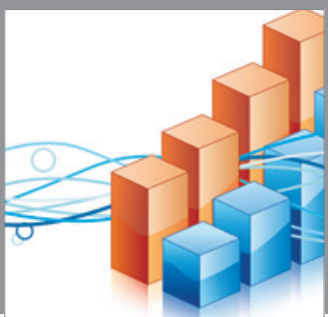

Advances in

Operations Research

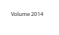

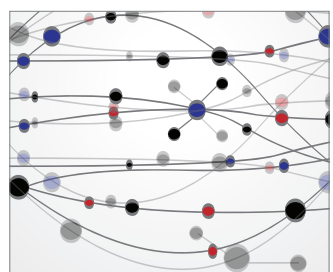

\section{The Scientific} World Journal
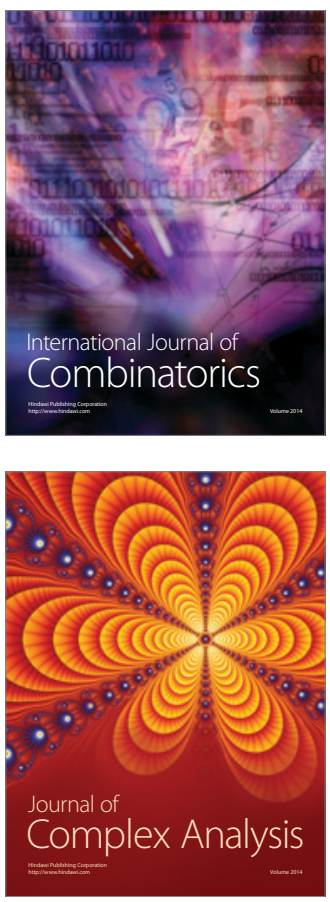

International Journal of

Mathematics and

Mathematical

Sciences
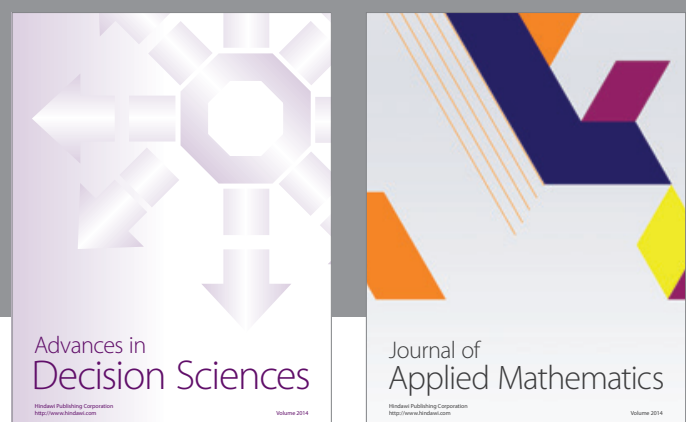

Journal of

Applied Mathematics
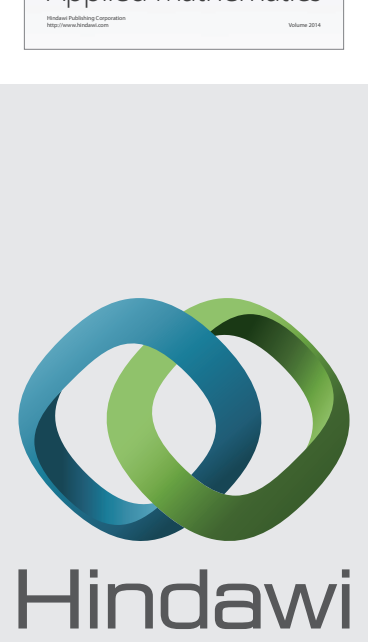

Submit your manuscripts at http://www.hindawi.com
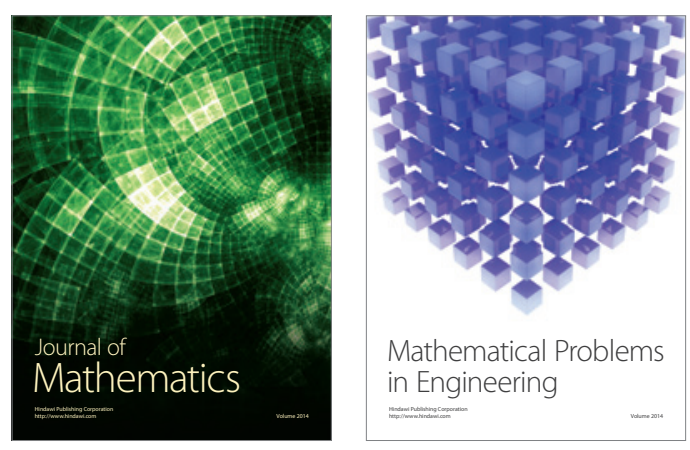

Mathematical Problems in Engineering
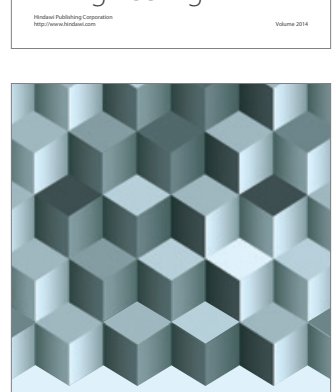

Journal of

Function Spaces
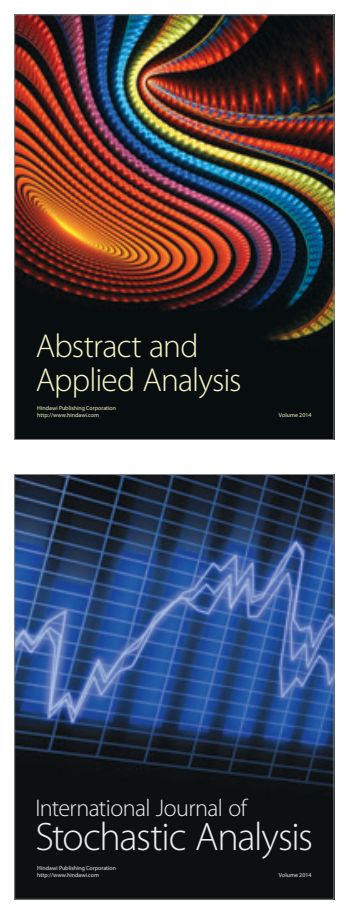

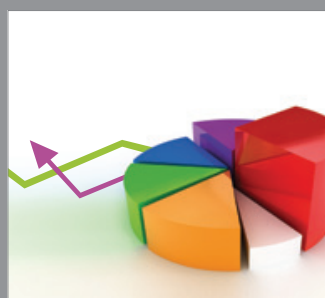

ournal of

Probability and Statistics

Promensencen
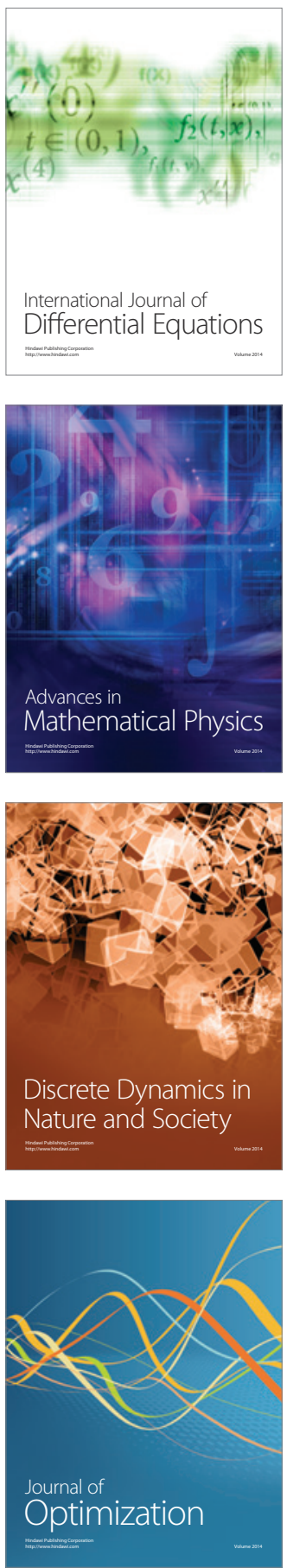